\title{
Linear Polarization and Magnetic Fields in Cool Stars
}

\author{
J. Huovelin ${ }^{1}$, S. H. Saar ${ }^{2}$ \\ ${ }^{1}$ Observatory and Astrophysics Laboratory, University of Helsinki, \\ Tähtitorninmäki, SF-00130 Helsinki, Finland \\ ${ }^{2}$ Harvard-Smithsonian Center for Astrophysics, Mail Stop 58, \\ 60 Garden Street, Cambridge, MA 02138, USA
}

\begin{abstract}
Observations and theoretical models of broadband linear polarization (BLP) in cool stars are discussed. Two effects, 1) differential saturation in magnetically sensitive absorption lines and, 2) Rayleigh and Thomson scattering are considered as the possible causes of the BLP. Results of theoretical models, and comparisons with observations are summarized.
\end{abstract}

\section{Theoretical models}

We have studied the expected rotational variations, wavelength dependence, and the absolute scale of broadband linear polarization for two mechanisms, 1) the magnetic intensification (MI), which is based on the cumulative effect of the differential saturation in the perpendicularly polarized Zeeman $(\pi$ and $\sigma$ ) components of spectral lines, and 2) scattering, either due to molecules (Rayleigh scattering), or electrons (Thomson scattering), in an optically thin medium (scattering optical thickness $\tau_{s} \leq 0.1$ ). The details of the models will be published elsewhere (Huovelin and Saar, 1991; Saar and Huovelin, 1991).

In both cases, the amount and distribution of surface inhomogeneities (in the magnetic field for MI, and surface brightness and $\tau_{s}$ for scattering) are very important factors in determining the total, surface integrated polarization (since polarization is a direction sensitive vector quantity). Therefore, unambiguous estimates on the expected levels of polarization cannot be made without independent knowledge on the distribution of the polarization inducers. In our models we have used Occam's razor and restricted the discussion to one region on the stellar surface. The advantage of this choice becomes clear considering more complex distributions, for which the total polarization can be derived by summing the polarization vectors for all individual regions. 
The polarization from scattering is directly related to the inhomogeneities in the optical thickness and the surface brightness. In the optically thin case, the linear polarization is actually proportional to the product $\tau_{s} \times I$ in a surface element. We have made estimates of the polarization assuming homogeneous brightness, and varying optical thickness. At present, we lack estimates of the optical depth variations due to inhomogeneities in stellar atmospheres. Therefore, we used the $\tau_{s}$ 's from the published homogeneous chromospheric models, and assumed that $\delta \tau_{s} \approx \tau_{s}$. The models predict a very small contribution from Thomson scattering in single F-K dwarfs, but in the extended envelopes of giants, supergiants, and close binaries, the effect may be significant. Rayleigh scattering from molecules and atoms also increases with decreasing gravity and temperature but, like Thomson scattering, is probably undetectable in cool dwarfs.

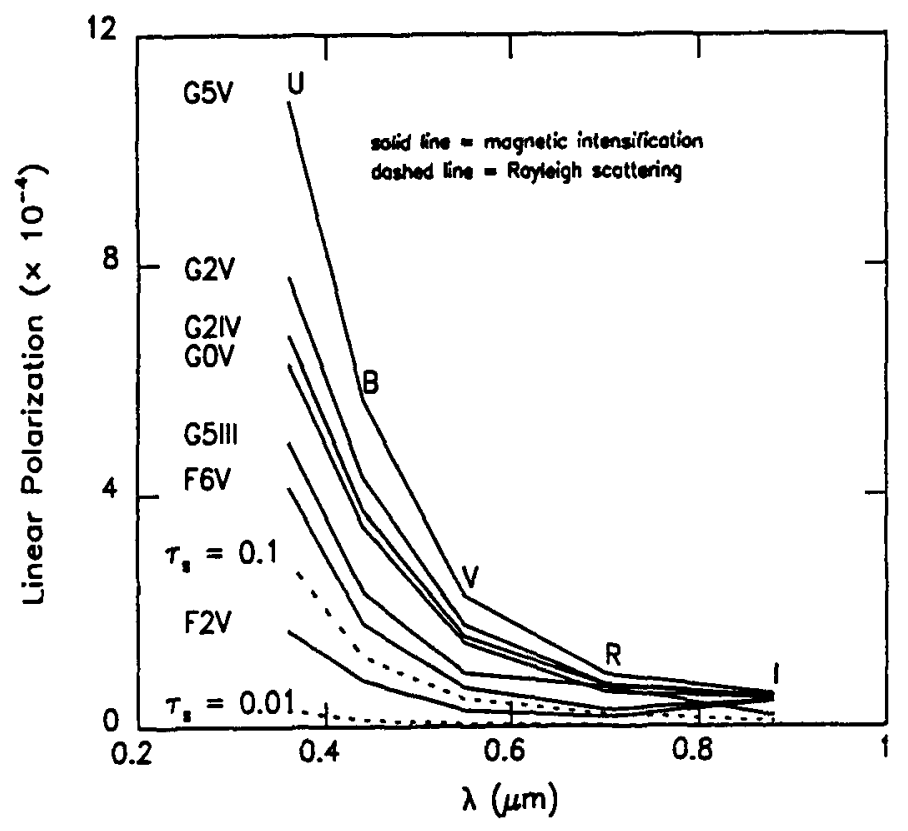

Fig. 1. Broadband linear polarization (maximum expected values with one circular region of size 24\%) in Johnson UBVRI passbands. The respective upper limits for Rayleigh scattering (size $18 \%$ ) are presented for comparison. $\tau_{s}=0.1$ corresponds roughly to values expected in early $\mathrm{K}$ giants, and $\tau_{s}=0.01$ in $\mathrm{K}$ dwarfs.

The models predict larger contribution from the differential saturation in dwarf stars, and the BLP could therefore be a direct indicator of magnetic activity in those stars. The time variations and the wavelength dependence are modelled for different spectral types for comparison with observations. The derived levels of BLP are low ( $\leq 0.1 \%$ in F-G dwarfs), and therefore difficult to measure. Fig.1 shows the theoretically expected broadband polarization for various spectral types in the Johnson UBVRI passbands. 


\section{Comparison with observations}

The present observational material does not contradict the theoretical predictions. The observations show signs of stellar polarimetric variability, but the amplitudes are small, and in most cases it is impossible to firmly distinguish the details (see e.g. Huovelin et al., 1989). Nevertheless, with simultaneous monitoring of other activity indicators (e.g. Ca-emission, photometry; Huovelin et al., 1987, 1988), the polarimetry may be used as an additional constraint.
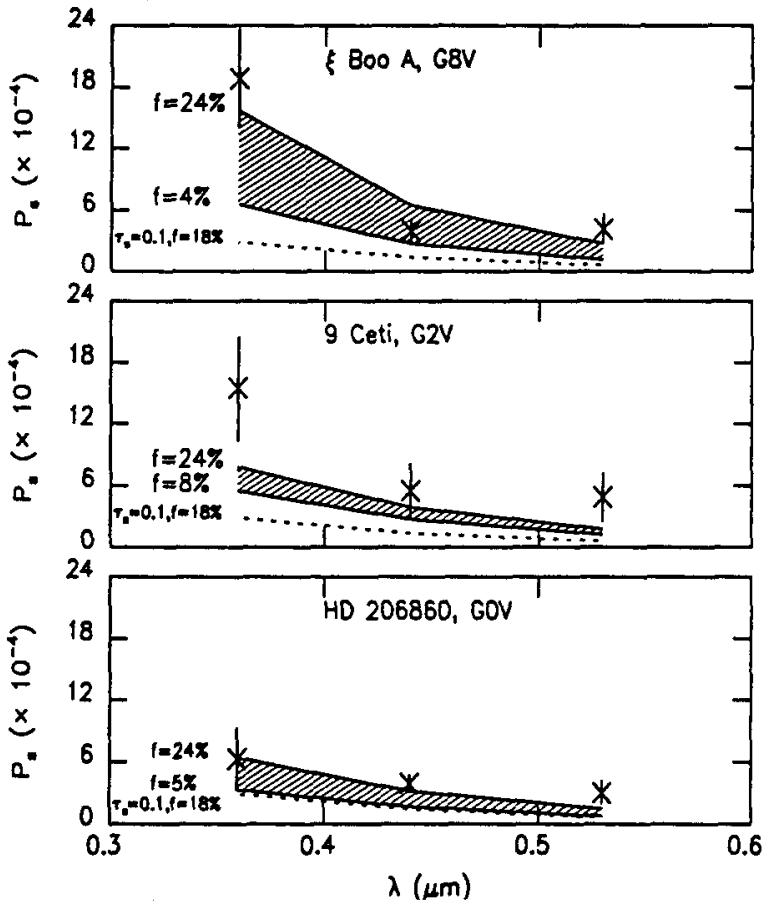

Fig. 2. Polarimetric observations in UBV (Huovelin et al., 1988) compared with models for differential saturation (shaded area) and the upper limit for Rayleigh scattering (dashed line). The error bars are all $3 \sigma$, and $f$ is the surface coverage of the region. Note, that our predictions of $\tau_{s}$ for dwarf stars are considerably lower than the upper limit (Saar and Huovelin, 1991).

The polarization is the largest in the ultraviolet, as expected on the theoretical grounds. Unfortunately, the observations in the ultraviolet are less accurate than in the longer wavelengths. A straightforward comparison of the BLP between different passbands may yield slopes for $P(\lambda)$ that are too steep, since the increase of random errors tends to increase also the average polarization, perhaps more than linearly with the errors. Fig. 2 gives a few examples of our comparisons between the observed and the theoretically expected polarizations. In Fig. 2 we also show indicative estimates of the surface coverages of magnetic fields, assuming that the polarization is due to the differential saturation. The complete tables of these estimates are given in Saar and Huovelin (1991). 


\section{Discussion}

The polarimetric models predict specific variations with time and with wavelength, which set a great challenge to the observations. In principle, the measurements of the time variations could yield very detailed information on the inhomogeneities and magnetic fields on the stellar surface, and the source of the polarization could be unveiled by studying the variations with wavelength.

However, the accuracy (a few times $10^{-5}$ ) required by quantitative tests of the theoretical models is not easy to achieve with current polarimeters. In addition to good photon statistics (i.e., bright stars and a big telescope), the observations have to be carried out with extreme care to avoid other random errors which can easily grow to a level higher than the expected polarization. The problem is most serious in the ultraviolet, where the photon statistics are lowest (cool stars are not very bright in the UV). Also, the atmospheric dispersion may cause substantial decentering of the UV stellar image in the diaphragm, which is not seen by visual inspection. The polarization caused by the edge of a metallic diaphragm, for example, may also be a serious problem in instruments where this effect has not been accounted for in the observing and reduction procedures. Finally, the differences in the wavelength dependence are largely smoothed out in the broadband measurements. Narrower passbands would be better in this sense, but have a decreased photon statistics, which limits the observations to very bright stars and/or big telescopes.

\section{References}

Huovelin, J., Piirola, V., Vilhu, O., Efimov, Yu., Shakhovskoy, N.M.: 1987, Astron. Astrophys. 176, 83

Huovelin, J., Saar, S., Tuominen, I.: 1988, Astrophys. J. 329, 882

Huovelin, J., Linnaluoto, S., Tuominen, I., Virtanen, H.: 1989, Astron. Astrophys. Suppl. Ser 78, 129

Huovelin, J., Saar, S.: 1991, Astrophys. J., in press

Saar, S., Huovelin, J.: 1991, Astrophys. J., submitted 